\title{
Tendencias del estudio Las prácticas de enseñanza de los docentes de educación secundaria
}

\section{Trends from the research Teaching practices of secondary education teachers}

Horacio Ademar Ferreyra ${ }^{1}$

Ana Rúa²

\section{Resumen}

Entre 2016 y 2018, los integrantes del Equipo de Investigación de Educación de Adolescentes y Jóvenes de la Facultad de Educación de la Universidad Católica de Córdoba (UCC), Unidad Asociada del Consejo Nacional de Investigaciones Científicas y Técnicas (CONICET), asumimos la tarea de concretar la investigación Las prácticas de enseñanza de los docentes de educación secundaria. Un estudio en las provincias de Córdoba, Buenos Aires y Entre Ríos, República Argentina.

Completado el período de indagación, presentamos este artículo cuyos propósitos son los siguientes:

- reseñar los veintidós documentos producidos agrupados en cuatro tipos que se corresponden con las etapas de desarrollo de la investigación: definiciones conceptuales y metodológicas, operativización de componentes, recolección y sistematización de evidencia empírica y comunicación de resultados;

\footnotetext{
${ }^{1}$ Doctor en Educación y Licenciado en Ciencias de la Educación, Universidad Católica de Córdoba; profesor titular e investigador principal (Unidad Asociada CONICET) en esa Universidad y de la Universidad Nacional de Villa María (Cat. 2), director del estudio que este artículo reseña. Córdoba, Argentina. Correo electrónico: dr.horacio.ferreyra@gmail.com.

2 Magíster de la Universidad de Buenos Aires en Didáctica. Licenciada en Educación. Profesora adjunta en la Universidad Nacional de Quilmes e investigadora invitada en el estudio cuyas tendencias se presentan. Buenos Aires, Argentina. Correo electrónico: arua@uvq.edu.ar.
}

Diälogas Pedagágicas - ISSN en línea: 2524-9274.

Año XIX, No 37, abril-septiembre 2021. Pág. 76-95. DOI: http://dx.doi.org/10.22529/dp.2021.19(37)06

Recibido: 17-10-2020 / Aprobado: 27-03-2021.

Artículo publicado bajo Licencia Creative Commons Atribución-NoComercial-SinDerivar. (c) Universidad Católica de Córdoba. 
- $\quad$ plantear información de síntesis que pueda ser profundizada y rastreada en los distintos documentos;

- presentar algunas de las tendencias que se han manifestado con más contundencia durante el estudio a partir del entrecruzamiento y triangulación de los datos obtenidos.

Palabras clave: escuela secundaria polivalente, enseñanza, didáctica.

\section{Summary}

Between 2016 and 2018, the members of the Research Team on Education for Adolescents and Young People of the Faculty of Education of the Catholic University of Córdoba (UCC), Associated Unit of the National Council for Scientific and Technical Research (CONICET), took on the task of carrying out the research Teaching practices of secondary education teachers. A study in the provinces of Córdoba, Buenos Aires, and Entre Ríos, Argentine Republic.

Having completed the period of investigation, we present this article whose purposes are the following:

- review the twenty-two documents produced grouped into four types that correspond to the research development stages: conceptual and methodological definitions, implementation of components, collection and systematization of empirical evidence, and communication of results;

- propose summary information that can be further developed and traced in the different documents;

- $\quad$ present some of the trends that have emerged more strongly during the research based on the cross-referencing and triangulation of the data obtained.

Keywords: high school, teaching, didactics.

La investigación Las prácticas de enseñanza de los docentes de educación secundaria. Un estudio en las provincias de Córdoba, Buenos Aires y Entre Ríos, República Argentina fue desarrollada entre 2016 y 2018 por los integrantes ${ }^{3}$ del Equipo de Investigación de Educación de Adolescentes y Jóvenes de la Facultad de Educación de la Universidad Católica de Córdoba (UCC), Unidad Asociada del CONICET.

En este artículo, se presentan los documentos producidos y las tendencias reconstruidas durante el estudio a partir del entrecruzamiento y triangulación de los datos obtenidos.

\footnotetext{
${ }^{3}$ Optamos por usar esta expresión para abarcar a los sujetos en general; no se debe a un sesgo sexista sino una preocupación por allanar la escritura y la lectura.
} 


\section{Los documentos que presentan la información recogida}

Los componentes globales de la investigación, tanto teóricos como metodológicos, están planteados en tres documentos:

Documento 1: Plan de trabajo (Ferreyra y Di Francesco, 2015). En este primer escrito, explicitamos los antecedentes del estudio sobre las prácticas de enseñanza en nuestro país y en el mundo y el estado de desarrollo alcanzado por nuestro equipo en la indagación de este campo de problemas. Expresamos las hipótesis que las prácticas de enseñanza desencadenan y los objetivos generales derivados de estas. Precisamos los acuerdos metodológicos para recabar datos de realidad y cómo esta información va a ser transformada en pruebas para contrastar con nuestras primeras conjeturas con el fin de respaldarlas o desecharlas. Construimos un plan de actividades con plazos de operativización. Identificamos cuál es el impacto previsto y cuáles son las estrategias de comunicación de los resultados que se irán obteniendo. Detallamos quiénes integran el equipo de investigación y cuáles son las funciones de cada miembro. Enunciamos la bibliografía disponible para un primer rastreo conceptual de la problemática y, como síntesis de todos estos componentes del estudio a encarar, anexamos la ficha técnica del proyecto.

Documento 2: Conceptos que dan marco a esta investigación (2016 a). En él, definimos las prácticas de enseñanza como: "... acciones conscientes, concretas, que se desarrollan en el contexto de las instituciones educativas y que implican situaciones de interacción con otras personas; están determinadas por una teoría que les da sentido y se sustentan en principios éticos que definen su intención: ayudar, del mejor modo posible, a que un estudiante aprenda y, de este modo, comprenda qué sucede en su realidad" (p. 5). Diferenciamos prácticas de enseñanza de acciones de enseñanza. Caracterizamos los rasgos centrales de las prácticas de enseñanza en función de tres dimensiones ${ }^{4}$. Precisamos aspectos que nos permiten entramar las prácticas de enseñanza con las prácticas educativas y sociales y con sus contextos de realización. Para completar esta teoría, definimos el concepto central de $\operatorname{modos}^{5}$, que permite agrupar las prácticas que el docente desarrolla cuando enseña.

Documento 3: Dimensiones, categorías y aspectos a estudiar (2016 b). Considerando los documentos precedentes, derivamos los componentes de las prácticas

${ }^{4}$ A partir de la conceptualización de práctica docente, decidimos abarcar las siguientes dimensiones:

"dimensión didáctica, que estudia qué tipo de previsiones y de acciones lleva adelante el docente en sus clases,

dimensión teórica, que investiga por qué el educador planificó de esa manera su tarea y cuáles son sus fundamentos para desarrollar esas acciones al enseñar, cuáles son sus supuestos y convicciones respecto de su actuación en la clase y de la enseñanza en general y

dimensión ética, que indaga qué es, para ese profesional, una buena enseñanza y cuál es la finalidad de las propias acciones en función de este compromiso por realizar su tarea del mejor modo posible, acortando la distancia entre lo que hace y lo que considera que sería correcto hacer" (p. 5). Aun reconociendo que la dimensión ética es también una dimensión teórica, optamos por diferenciar una de otra para destacar la decisiva importancia de los componentes valorativos -abarcados en la primera- en las prácticas de enseñanza.

${ }^{5}$ Distinguimos: "... modos -matrices, modelizaciones...- que van a permitir agrupar las prácticas de enseñanza pero que no se confunden con ellas, que son siempre distintas, personales, singulares (...) La idea de modo de enseñar posibilita clasificar la diversidad frondosa que se va registrando en las observaciones y en las entrevistas realizadas, y sistematizar sus rasgos más elocuentes, sus manifestaciones más estables; posibilita organizar los datos y, a la vez, la visibilización de emergentes; ayuda a enfocar características clave pero siempre considerando que más allá de este encuadre simplificador existe una realidad compleja y densa" (p. 13). 
docentes en los que centramos nuestra investigación -categorías y aspectos que componen cada dimensión- y optamos por aquellos de mayor potencialidad explicativa.

A partir de estos documentos, generamos un segundo tipo de escritos destinados a aunar las estrategias de intervención de los setenta y tres investigadores y de los ciento seis investigadores en formación involucrados en el estudio.

Documento 4: Planificación de la encuesta virtual (2016 c). Este texto se conforma de los intercambios realizados por los integrantes del Equipo de Investigación de Educación de Adolescentes y Jóvenes acerca de los aspectos a presentar en la encuesta virtual y de la formulación de cada uno de ellos de modo tal que el instrumento permita la obtención de datos confiables.

Documento 5: Guía para la observación de clases (2016 d). Análogamente al anterior, este documento sintetiza los aspectos a ser observados en cada clase de educación secundaria considerada y los recaudos metodológicos que los procesos de registro y de transcripción involucran.

Las memorias de las reuniones del equipo de investigadores y de las tareas que se realizan son documentos de circulación interna que también se incluyen en este grupo.

A medida que concretamos las instancias previstas para la obtención de datos -encuestas en línea, observación de clases, entrevistas con los docentes, análisis de planificaciones, encuentros de trabajo con los educadores cuyas clases han sido incluidas en el estudio, foros presenciales y virtuales, consultas previas y posteriores a cada uno de estos foros, consulta a investigadores, reunión de grupos focales, convocatorias para la presentación de relatos, ensayos, secuencias didácticas, fotografías y videos-, va conformándose un tercer grupo de documentos que sistematiza la base empírica recogida.

Documento 6: Las prácticas de enseñanza de los docentes de Educación Secundaria a través de encuestas (2019 a). En esta publicación, presentamos los resultados de la encuesta en línea implementada durante junio de 2017, que fue respondida por 2819 docentes. La información recogida nos permite identificar regularidades respecto de cómo esos educadores caracterizan sus prácticas de enseñanza al inquirir acerca de veinticuatro aspectos que abarcan la planificación, la enseñanza y la evaluación de aprendizajes. Los datos que la encuesta provee nos permiten identificar tendencias auspiciosas para la dimensión teórica de nuestro estudio -aquella que indaga los saberes declarativos de los docentes, sus ideas- y, también, detectar evidencias que plantean alertas didácticas. De unas y de otras, no solo destacamos porcentajes de presencia y de ausencia, sino testimonios que, por su riqueza, operan como estampas de situación' (Stake, 2006, p. 149).

Documento 7: Las prácticas de enseñanza de los docentes de educación secundaria a través de observaciones de clases, planificaciones didácticas y entrevistas (2019 b). Sistematizamos la observación de 2295 clases en función de diecisiete categorías: inicio de los intercambios entre docente y estudiantes, movilización de los recursos cognitivos de quienes aprenden, provisión de nuevos contenidos, planteamiento de

\footnotetext{
${ }^{6}$ Las estampas incluidas en nuestra investigación son testimonios breves que codifican analizadores clave para la comprensión de las prácticas de enseñanza; son elocuentes, densas y ayudan a caracterizar no solo el pensamiento o la acción de un docente en particular, sino de todo un grupo de colegas.
} 
actividades, promoción de interacciones, entre otras. Para complementar los datos obtenidos a través de este proceso de observación, presentamos nuestro análisis de 2006 planificaciones de asignatura y de clase de estos docentes y los rasgos centrales surgidos de las entrevistas que efectuamos a 2111 educadores.

Documento 8: Segundo Foro y Seminario. Debates sobre el currículum de Ciencias Naturales de la educación secundaria en relación con las prácticas: avances, retos y desafíos de cara al futuro (2018 a). Además de la encuesta general destinada a todos los docentes abarcados en el estudio, implementamos una segunda consulta, también en línea, dirigida a grupos de profesores de un campo de contenidos específico. Los resultados de la consulta a profesores de Ciencias Naturales están presentados en este documento, así como los intercambios concretados en el Segundo Foro y Seminario realizado el 21 y 22 de noviembre de 2016. En este foro, nos reunimos cincuenta y tres docentes e investigadores de este campo del conocimiento escolar y analizamos los datos surgidos de la encuesta en distintas mesas de trabajo focalizadas en: Prácticas de enseñanza de las Ciencias Naturales en la Educación Secundaria, Planificación de las prácticas de enseñanza, Gestión de la enseñanza, Prácticas de evaluación, Los buenos profesores de Ciencias Naturales y Las mejores clases de Ciencias Naturales. Esta publicación también incluye ideas centrales de las conferencias de investigadores invitados: Jorge Bartolucci (Universidad Nacional Autónoma de México), Melina Furman (Universidad de San Andrés e Instituto Nacional de Formación Docente), Hernán Miguel (Universidad de Buenos Aires) y Hugo Labate (Ministerio de Educación y Deportes de la Nación), cuyos videos están disponibles en la página web de la universidad. También, reseñamos los intercambios que estas presentaciones generan en los profesores participantes.

Documento 9: Debates sobre el currículum de Ciencias Sociales y Humanidades en la educación secundaria, en clave de las prácticas educativas que miran los tiempos presentes y por-venir. Informe final (2018 b). Con una estructura equivalente a la del documento anterior, en esta publicación, sistematizamos los resultados de la consulta en línea realizada a los docentes de Ciencias Sociales involucrados en el estudio y los testimonios de sesenta de ellos que, también, asisten al foro presencial realizado el 11 y 12 de mayo de 2017. Además, socializamos los planteos expresados por los investigadores invitados: Cecilia Cresta y Alicia Graciela Funes (Universidad Nacional del Comahue), Eduardo Corsi (Universidad Nacional de Córdoba), Daniel Bargman (Ministerio de Educación y Deportes de la Nación), Patricia Romero (exfuncionaria del Ministerio de Educación de Córdoba) y Celeste Cerdá (Universidad Nacional de Córdoba), así como los vínculos establecidos por los asistentes entre estas ideas y sus prácticas de enseñanza en escuelas secundarias.

Documento 10: Debates sobre el currículum de Matemática en la educación secundaria: avances y desafíos en relación con las prácticas de enseñanza, de cara al futuro. Informe final (2018 c). Formando una unidad con las dos publicaciones anteriores, esta reseña la convocatoria en línea a "registrar ideas de la enseñanza de la Matemática en la educación secundaria respecto de tensiones, afirmaciones y problemáticas que exploren la planificación, gestión y evaluación" (p. 8) a la que son invitados todos los docentes de este campo de contenidos implicados en la investigación. Sesenta y tres de ellos, además, participan en el foro presencial del 22 y 23 de mayo de 2017 para: "Analizar las prácticas de enseñanza de la Matemática en la educación secundaria de hoy con proyección al futuro" (p. 10), intercambios que sistematizamos en esta publicación. El documento, también, socializa los aportes de 
los investigadores: Hugo Labate (Ministerio de Educación y Deportes de la Nación), Esther Galina (Ministerio de Ciencia y Tecnología de la provincia de Córdoba), Cristina Esteley y Mónica Villarreal (Facultad de Matemática, Astronomía, Física y Computación de la Universidad Nacional de Córdoba), Gema Fioriti y Rosa Ana Ferragina (Centro de Estudios en Didácticas Específicas de la Universidad Nacional de San Martín), así como las ideas que desencadenan en los docentes participantes.

Documento 11: ¿Qué es lo que ocurre en nuestras aulas de secundaria? Ensayos referidos a la educación secundaria argentina (2017 a). Ochenta y siete educadores e investigadores participan de esta convocatoria de nuestro equipo a través del aporte de ensayos "-género permeable a la diversidad de perspectivas y modos de decir-, vehículo óptimo para revelar y develar lo que sucede en cada escuela y en cada aula" (p. 10). Treinta de las obras son seleccionadas para formar parte de esta publicación, enlazadas por un prólogo que sintetiza los rasgos clave detectados en los ensayos respecto de las prácticas de enseñanza, entre otras: cómo se piensa y se concreta la inclusión, qué actitudes se mantienen y deponen en la tarea cotidiana y cuáles son los compromisos que movilizan a educadores y a estudiantes.

Documento 12: Enseñar en educación secundaria, un compromiso con la construcción de aprendizajes relevantes y duraderos. Secuencias didácticas implementadas en los distintos ciclos y años de la educación secundaria y Modalidades (2019 c). Esta publicación recopila cuarenta y seis secuencias didácticas que fueron presentadas por docentes de educación secundaria como respuesta a la convocatoria motorizada por nuestro equipo de investigación. Geometría en la Danza Clásica, Inventariado y catalogación de bienes culturales, La videopoesía como método de experimentación y resignificación, Los juegos tradicionales y Las unidades de medida a través de la imagen son solo algunos de los nombres de esas propuestas de enseñanza. Cada una de ellas aporta datos esclarecedores acerca de la tarea en las aulas de este grupo de docentes innovadores, ya que uno de nuestros requerimientos fue que las propuestas hubieran sido implementadas en el aula y que estuvieran acompañadas por producciones de los estudiantes.

Documento 13: Educación secundaria: Prácticas de enseñanza en foco (2017 c). Presenta fotografías y videos referidos a la educación secundaria surgidos de la convocatoria de la UCC a los docentes abarcados en el estudio y a estudiantes de escuela secundaria. Las obras están agrupadas en tres focos: La realidad, El contraste, El porvenir y son acompañadas por textos breves elaborados por integrantes de nuestro equipo de investigadores.

Documento 14: Dimensión ética (2018 d). En un momento de la tarea, los profesionales involucrados en la sistematización de la información advertimos que no estábamos contando con información concluyente para profundizar en la dimensión ética del estudio, por lo que resultaba necesario acceder a testimonios que permitieran triangular las tendencias que iban surgiendo a fin de validarlas. Entonces, optamos por organizar grupos focales en los que los docentes expresaran su posicionamiento ético: qué es una buena enseñanza, cuáles son los motivos de esta caracterización y cómo se vincula con la acción didáctica que realiza. Esta publicación de circulación interna reseña las discusiones llevadas adelante en esos grupos por los ciento doce docentes convocados.

Documento 15: Ventanas de Johari sobre prácticas docentes (2018 e). Con la misma finalidad que los grupos focales -confirmar tendencias que iban detectándose 
en la base empírica obtenida-, solicitamos a cuatrocientos quince docentes abarcados en el estudio que plantearan respuestas breves a cuatro preguntas interconectadas presentadas a modo de ventana: características que tiene tu manera de enseñar y que no quisieras que cambien (¿Con qué estás conforme?), características que tiene tu manera de enseñar y que quisieras que cambien (¿Qué le sobra?), características que no tiene tu manera de enseñar y que no quisieras adquirir, características que no tiene tu manera de enseñar y que quisieras incorporar (¿Qué le falta?). Este documento de circulación interna integra y sistematiza la información provista.

Finalmente, completando los documentos que describen el proyecto de investigación, los que reseñan los acuerdos instrumentales a los que llegan los integrantes del equipo y los que presentan integraciones de los datos de realidad obtenidos, desarrollamos un cuarto grupo de escritos para dar a conocer, sucesivamente, primeras tendencias y conclusiones avanzadas del estudio. Así, realizamos un proceso de socialización, difusión y divulgación en el colectivo educativo y en la comunidad social, que responde al principio de Proyección y responsabilidad social de nuestra universidad (UCC, 2011). Hasta este momento, marzo de 2019, hemos presentado siete documentos que cumplen con esta función y este informe final es el octavo.

Documento 16: Dimensiones involucradas en el estudio de las prácticas de enseñanza (2018 f). En este artículo, publicado por la Universidad Simón Bolívar de Colombia, planteamos el marco teórico de la investigación a la comunidad académica.

Documento 17: Las prácticas de enseñanza como dimensión constitutiva de la calidad de la educación (2018 g). También dirigido a la comunidad académica, este artículo ha sido publicado por la Universidad de Babahoyo, Ecuador.

Documento 18: Gacetillas de prensa (2018 h). Cuando fuimos obteniendo los primeros resultados estables de la investigación, los consolidamos -para su difusión- a través de medios de comunicación masiva. Una de estas gacetillas es publicada, por ejemplo, por el periódico La Voz de Córdoba (Otero, 2018).

Documento 19: Mirar para aprender en contexto. Observación de clases (2017 b). Este documento opera como primera devolución de nuestro equipo a los docentes y a las instituciones que colaboraron con el estudio al permitir que sus clases fueran observadas. Presenta las tendencias iniciales señaladas por los investigadores respecto de la dimensión didáctica, la que hace foco en las tareas de programación didáctica y en las acciones de enseñanza.

Documento 20: Rueda del aprendizaje escolar. Recurso didáctico (2018 i). También como retroalimentación a los docentes e instituciones participantes y con el propósito de socializarlo a las instituciones formadoras de educadores, desde nuestro Equipo de Investigación de Educación de Adolescentes y Jóvenes, generamos un recurso didáctico que ayuda a enfocar el aprendizaje de los estudiantes con "una visión integradora (...) con una perspectiva reconciliadora, que no opere excluyendo autores, posicionamientos e interpretaciones, sino que proceda tratando de encontrar puntos de diálogo" (p. 6) entre las distintas teorías del aprendizaje.

Documento 21: Las prácticas de enseñanza en la educación secundaria I (2019 d). Con el mismo compromiso de proyección y responsabilidad social universitaria, en junio y noviembre de 2018, desarrollamos dos encuentros que reunieron a docentes proveedores de testimonios para esta investigación. En la jornada del 27 de junio, 
participan noventa y ocho profesores entre los colegas que aportan relatos, ensayos, secuencias didácticas, fotografías o videos. Con ellos, analizamos propuestas socializadas por los mismos pares docentes, cuestiones focales referidas a las prácticas de enseñanza y se realizó la presentación de resultados en la conferencia Tres tendencias generativas registradas en el estudio de las prácticas de enseñanza. La investigadora Nora Alterman (Escuela de Ciencias de la Educación de la Facultad de Filosofía y Humanidades, Universidad Nacional de Córdoba) cierra el encuentro. Los testimonios expresados en la consulta previa y socializados en las comisiones, así como los videos que registran las dos presentaciones, forman parte de esta publicación.

Documento 22: Las prácticas de enseñanza en la educación secundaria II (2019 e). El 6 de noviembre de 2018, concretamos el segundo encuentro. En esta ocasión, son convocados sesenta y cuatro docentes cuyas clases han sido presenciadas por el equipo de investigadores, quienes también forman parte del intercambio. A lo largo de la jornada, el grupo considera la presentación Tendencias detectadas a través de la observación de clases, la que se completa con talleres simultáneos de discusión y proposición por áreas de contenidos. También, se integra la conferencia Debates sobre el currículum de enseñanza secundaria: poniendo el foco en la evaluación, que socializa el próximo proyecto de investigación del equipo de la UCC y a partir de la que se desarrollan talleres centrados en la evaluación de los aprendizajes.

Hasta aquí, la reseña de las publicaciones que surgen de la sistematización de las evidencias, así como del trabajo de preparación para obtenerlas, organizarlas y comunicarlas. En estos escritos, se encuentra la información detallada acerca de cada una de las dimensiones, categorías y aspectos considerados en Las prácticas de enseñanza de los docentes de educación secundaria (...). Recomendamos su lectura para tener una comprensión profunda del campo indagado, dado que las páginas que siguen presentan ideas que hemos seleccionado para este artículo, pero no todas las registradas.

\section{Algunas tendencias destacables}

En esta segunda parte, reseñamos algunas de las tendencias registradas en el estudio. Optamos por denominarlas tendencias y no conclusiones al entenderlas como conocimientos "plausibles" (Becker, 2018) respecto de las prácticas de enseñanza. Mientras las conclusiones demostrativas están más allá de las controversias y son definitivas, las conclusiones plausibles son provisorias (p. 25), con potencial para seguir produciendo conocimiento a partir de ellas dada su condición de inacabadas.

Compartimos, entonces, los productos de nuestro estudio agrupados en:

a. Tendencias generales respecto de los modos de enseñar.

b. Tendencias más específicas.

C. Tendencias emergentes.

d. Tendencias que se presentan como desafíos a asumir. 


\section{a. Tendencias generales respecto de los modos de enseñar}

En la investigación que asumimos, nuestro punto de partida es la gran categoría conceptual modos de enseñar en la que está focalizado el objetivo general del estudio: "Analizar los modos a través de los cuales los docentes configuran las prácticas de enseñanza en instituciones educativas de educación secundaria (...)" (2015, p. 17).

Respecto de estos modos de enseñar, hemos detectado cuatro tendencias generales:

Tendencia 1: Alto involucramiento didáctico de los educadores

Los datos nos permiten destacar un claro compromiso profesional respecto de la tarea de transmisión de contenidos de una generación a otra que se concreta en la escuela. El tiempo de las clases se centra en este principio que, por supuesto, no confundimos con la exposición docente ni con una clase magistral, sino que remite a la asunción continua de la tarea de enseñar para que los estudiantes aprendan, con la responsabilidad de un sujeto adulto para con quienes están comprendiendo su realidad de otra manera a partir de la acción escolar.

En simultáneo con este rasgo académico, registramos lo siguiente:

Tendencia 2: Cercanía afectiva de los profesores con los estudiantes

Predominan los vínculos personales de amabilidad, respeto y disponibilidad hacia quienes aprenden. También, se advierte con contundencia el registro de los educadores de lo que sucede con quienes componen sus clases, más allá de los saberes previstos: su interés por comprender a los estudiantes no solo como tales, sino como personas sociales completas.

Estos dos rasgos positivos -compromiso con la enseñanza y afecto por los estudiantes- detectados en nuestra investigación se enlazan con dos rasgos menos auspiciosos en las prácticas estudiadas. Aquí, los enunciamos en términos generales, ya que, más adelante -en la expresión de otras tendencias más específicas-, nos ocupamos de desagregarlos.

Tendencia 3: Preeminencia de la "teoría de la huella" (Meirieu, 2016, p. 11)

Esta concepción de la enseñanza que estamos destacando con el propósito de promover un alerta respecto de su preponderancia ${ }^{7}$ sostiene que es suficiente exponer un contenido a los estudiantes para "provocar" un aprendizaje. Los resabios tradicionales en la metodología de enseñanza, es decir, su integración en un número importante de clases como práctica de enseñanza excluyente resultan preocupantes porque reflejan un posicionamiento distorsionado respecto de cómo aprenden los estudiantes. Aun cuando en las encuestas y en otros instrumentos de recolección de información declarativa los docentes expresan que la prioridad de la enseñanza está puesta en la

\footnotetext{
7 En nuestra investigación (2019 b), detectamos que un $60 \%$ de las clases de Ciencias Sociales son tradicionales y a estas se suma un $6 \%$ de clases conductistas/tecnicistas -aquellas estructuradas en función de secuencias estímulo-respuesta-refuerzo-estímulo-respuesta- refuerzo...- en las que también se enfatiza la "teoría de la huella", pero ya no respecto de conceptos, sino de la adquisición de comportamientos técnicos, repetitivos, automatizables. En el campo de las Ciencias Naturales, 58 \% de las clases corresponde a la enseñanza tradicional y $19 \%$, a la conductista. En las clases de Lenguajes y Comunicación, las proporciones que representan la metodología implementada son, respectivamente, del $14 \%$ y del $29 \%$. En Matemática, las prácticas docentes tradicionales se registran en $7 \%$ de las clases y las conductistas, en $84 \%$.
} 
construcción de capacidades, la observación de clases da cuenta de que aún persiste una opción metodológica basada en la presentación de ideas y de conductas que insta al almacenamiento literal y a la repetición de contenidos por parte de quienes aprenden, más que a su comprensión y a su transferencia a nuevas situaciones. Este rasgo de promoción de huellas también se vincula con el predominio tradicional en las formas de evaluación.

Hacemos aquí un paréntesis en la enumeración: las observaciones de clase en conjunción con las entrevistas nos han resultado instrumentos privilegiados para el conocimiento al ayudarnos a confrontar lo que los docentes sostienen conceptualmente y lo que hacen. Hemos podido comparar los dos corpus de información: la reflexión docente (en nuestra investigación, la dimensión teórica) y sus acciones (la dimensión didáctica) y de esta contrastación surge la siguiente tendencia.

Tendencia 4: Desajustes entre lo expresado respecto del modo de enseñar y lo observado en las clases

Este rasgo reviste una particular importancia, dado que -entre los planteos del marco teórico de nuestra investigación (2016 a)- se encuentra la revisión de toda forma de "desajuste, elisión e ilusión" (tomamos la expresión original de Kemmis, 1996, p. 34) al enfocar el estudio de las prácticas docentes. Entonces, en este momento de establecer las tendencias que hemos conformado en estos tres años de estudio, resulta importante destacar este desajuste en los modos de enseñar que se detecta entre las ideas de los profesores acerca de una buena enseñanza y su acción en el aula. Mientras en lo expresado por los docentes predomina el discurso socioconstructivista, en sus acciones se registran trazas tradicionales. Acercamos un ejemplo: el principio de "partir de los conocimientos de los estudiantes", expresado por una mayoría de educadores como descriptor para su modo de enseñar, opera más como enunciado que como auténtica práctica, ya que esos recursos cognitivos de quienes aprenden solo son integrados en un número pequeño de clases en las que se parte de ellos para ayudar a los estudiantes, a lo largo de la tarea, a redescribir esas primeras apreciaciones acerca de un problema.

Así, algunas de las tendencias auspiciosas señaladas en nuestra sistematización de encuestas, de foros, de grupos focales, de ventanas -todas centradas en el saber docente asertivo- se restringen al considerar cómo esas ideas son implementadas en las clases reales, donde se advierten vestigios de una enseñanza preocupada por la impresión de contenidos en quienes aprenden, más que por la promoción del pensamiento de los estudiantes.

Hasta aquí, las cuatro tendencias generales que hemos comprobado en nuestro estudio. A continuación, nos referimos a los rasgos más específicos de los modos de enseñar que también destacamos con nitidez en nuestra investigación.

\section{b. Tendencias más específicas}

Como componentes rotundos que dan cuenta de cómo se concretan esos modos de enseñanza -rasgos acompañados por porcentajes elevados-, detectamos la tendencia que se menciona a continuación.

Tendencia 5: Conocimiento de los diseños curriculares y su integración en las planificaciones de asignatura y de clase 
Los grandes estructurantes de los documentos jurisdiccionales -prioridades pedagógicas, contenidos, ejes, componentes transversales, aprendizajes a construir por los estudiantes, principios metodológicos, etc.- están presentes en las programaciones que los docentes elaboran.

Tendencia 6: Diseño de planificaciones claras

Leyendo las planificaciones de los docentes es posible saber cuál es la propuesta de ese educador para los estudiantes: son elocuentes. Además, son compactas; se advierte coherencia y cohesión en sus componentes: contenidos, objetivos, actividades/ metodología, recursos, evaluación.

Tendencia 7: Articulación de los distintos momentos de la clase

Con porcentajes destacados, las acciones de enseñanza encaradas constan de distintos momentos -inicio, desarrollo, cierre- convenientemente señalizados para guiar a los estudiantes respecto de las acciones que se espera de ellos en cada momento. Durante la etapa inicial, suelen recuperarse contenidos que se ahondan y expanden en el tramo de desarrollo y se sintetizan en la instancia de cierre.

Tendencia 8: Promoción de vínculos entre clases, así como entre contenidos abarcados en encuentros anteriores

Advertimos una superación contundente de la idea de una clase como islote, con contenidos cerrados en sí mismos. Como contracara, se detecta el esfuerzo de los docentes para que los estudiantes adviertan cómo se entrama una clase con otras, cómo cada reunión forma parte de un proceso de intercambios más amplio.

Tendencia 9: Provisión de ayudas a los estudiantes durante el proceso de construcción de aprendizajes

La casi totalidad de los docentes abarcados en nuestro estudio acerca ayudas a los estudiantes: ampliación de contenidos, profundizaciones, señalamiento de errores, sugerencias de cómo hacerlo mejor, focalización de recaudos a tener en cuenta. En unas líneas más, cuando consideremos algunas debilidades en las prácticas de enseñanza, vamos a retomar este reconocimiento de la buena disposición docente para asistir a quienes aprenden para detenernos a especificar cómo el profesional concreta estas ayudas porque, si bien esta dedicación que señalamos es un componente valioso que está presente en todas las clases, no parece resultar suficiente.

Tendencia 10: Integración de recursos didácticos

Porcentajes elevados de docentes (48 \% al $93 \%$, según el área curricular) proponen la consulta de libros, la realización de actividades en hojas de trabajo por ellos diseñadas, el visionado de películas y la búsqueda de información en entornos virtuales. Como sucede con la tendencia anterior, más adelante ahondamos en este rasgo porque la presencia de materiales, si bien es por completo auspiciosa, puede ser mejorada en un marco de ideas más actualizado respecto de cómo aprenden los estudiantes y cómo los recursos inciden en estos logros.

\section{c. Tendencias emergentes}

Hasta aquí, hemos puesto a consideración tendencias dominantes: más generales las primeras, más acotadas a un aspecto didáctico las segundas. A continuación, pre- 
sentamos rasgos positivos que, aun cuando en nuestros estudios no están acompañados por porcentajes mayoritarios, han crecido a lo largo del lapso en el que nuestra investigación se desenvuelve y respecto de estudios anteriores que hemos llevado a cabo (Ferreyra, 2015), por lo que resultan testimonios de cambio promisorios.

Tendencia 11: Inclusión de cuestiones de realidad con las cuales vincular los contenidos escolares

La preocupación de los docentes para que los contenidos de las clases se encuentren anclados en situaciones que los estudiantes experimentan en su vida cotidiana y la promoción de vínculos estrechos entre el saber escolar y los contenidos que necesitan quienes aprenden para comprender su realidad y transformarla resultan tendencias favorables aun cuando no se manifiestan con el mismo ímpetu en todas las áreas de contenidos y aun cuando este ingreso de la realidad al aula toma -incluso- un carácter más ilustrativo - otra vez la teoría de la huella- que problematizador. En este sentido, es importante destacar la disposición de los docentes para integrar situaciones vitales específicas de los jóvenes y adolescentes -o de las personas adultas, en escuelas secundarias con esta modalidad- dentro de estos hechos contextualizados que permiten que nos refiramos a una enseñanza y a un aprendizaje situados.

\section{Tendencia 12: Promoción del trabajo en equipo}

Los docentes instan a los estudiantes a reunirse con sus pares para la realización de las distintas tareas asignadas y propician intercambios más o menos exigentes ${ }^{8}$ : desde la búsqueda de información hasta la construcción de productos. Lo hacen con frecuencias distintas según las áreas de contenidos curriculares (de $32 \%$ a $92 \%$ ), pero aun cuando el piso de este rango nos remite a una presencia esporádica en algunas asignaturas, es constitutivo de muchas otras situaciones de enseñanza y de aprendizaje.

Tendencia 13: Socialización de criterios para la realización de tareas

También, se registran disparidades en las clases correspondientes a los distintos campos de contenidos, pero resulta importante señalar lo propicio de la práctica de comunicar a los estudiantes cuáles son los requerimientos para sus desempeños y producciones, los que han de retomarse al momento de ser evaluados.

Tendencia 14: Presencia de formatos curriculares distintos del de una asignatura convencional

Proyectos, laboratorios, talleres, clínicas, ateneos, seminarios y trabajos de campo todavía no se presentan como formas generalizadas para la organización de contenidos de las unidades didácticas o de las clases ni para la organización de las actividades de los estudiantes, pero su inclusión denota una extensión ampliada progresiva entre 2016 y 2018, años en los que se lleva adelante nuestra investigación.

\footnotetext{
${ }^{8}$ Las ideas de enseñanza y aprendizaje exigentes (Subsecretaría de Promoción de Igualdad y Calidad Educativa, 2011, pp. 25-27) permiten diferenciar entre:

- $\quad$ propuestas docentes que remiten a la reproducción -poco exigentes: instan a los estudiantes a recordar información o a realizar una técnica de acuerdo con un modelo dado-y

- $\quad$ propuestas comprometidas con la comprensión -tareas exigentes: promueven indagar en los componentes de un concepto (reconstructivas), ubicar ese concepto en un marco mayor de ideas (reconstructivas globales) o crear algo nuevo (constructivas)-.
} 
Tendencia 15: Conformación de equipos docentes

En los foros, encuestas, consultas previas a encuentros y grupos focales, los educadores valoran positivamente el trabajo de planificar en equipo y de organizar clases coordinadas en conjunto por varios docentes. Sin embargo, a través de los mismos canales, expresan que no siempre es posible tal concreción.

\section{d. Tendencias que se presentan como desafíos a asumir}

Finalmente, desde el Equipo de Investigación de Educación de Adolescentes y Jóvenes de la Facultad de Educación de la Universidad Católica de Córdoba a cargo de la investigación Las prácticas de enseñanza de los docentes de educación secundaria. Un estudio en las provincias de Córdoba, Buenos Aires y Entre Ríos, expresamos cuestiones en las que resultaría oportuno seguir trabajando. Por ejemplo, a través de acciones de formación continua para acompañar a los docentes en su superación.

Que estos aspectos a mejorar sean más que las fortalezas que presentamos hasta aquí no tendría que llevarnos a pensar en prácticas docentes en estado de emergencia, sino en que hemos hecho un esfuerzo por expresarlos de una manera muy precisa para que puedan ser considerados pormenorizadamente si se asume un compromiso de cambio.

Tendencia 16: Falta de centralidad de una metodología de enseñanza desencadenada a partir de problemas

Aun cuando se advierten esfuerzos docentes por comenzar las clases y secuencias didácticas que plantean problemas a los estudiantes para que estas situaciones a desentrañar den sentido a los contenidos a aprender, esta metodología socioconstructiva no es la dominante ${ }^{9}$. Así, los docentes no parecen asumir como propósito generalizado que los estudiantes adquieran capacidades vinculadas con la ciencia escolar -natural o social-, con el pensamiento matemático, con el cuidado del cuerpo y el ambiente, con el análisis o la producción de obras artísticas, tecnológicas o comunicativas, es decir, con aquellos quehaceres propios del campo de conocimientos que enseñan, sino que predomina una organización didáctica basada en la secuencia teoría-práctica. Los docentes proveen contenidos o asignan la lectura de materiales que los aportan y, luego, en algunos casos, los estudiantes los vinculan con cuestiones prácticas. En cambio, una metodología deseable promovida por las teorías del aprendizaje y por las teorías didácticas actuales está dada por una estructura práctica-teoría-práctica que no se detecta con preeminencia en nuestro estudio.

Tendencia 17: Preponderancia de resolución de ejercicios mediante la aplicación de algoritmos modelizados por el docente

Estrechamente vinculada con el rasgo anterior, advertimos un énfasis didáctico puesto en que los estudiantes dominen técnicas por sobre una enseñanza que los ayude a resolver problemas. No ha de descartarse que los educadores ayuden a los estudiantes a automatizar algunos aprendizajes, pero no es aceptable que la enseñanza se

\footnotetext{
${ }^{9}$ En el campo de las prácticas docentes en Lenguajes y Comunicación, se manifiesta en un 35 \% de las clases; en Ciencias Sociales, en un $32 \%$; en las clases de Ciencias Naturales, la opción por una metodología socioconstructivista es del $22 \%$ y en Matemática, del $7 \%$.
} 
reduzca a promover un adiestramiento a partir de un ejercicio-tipo, adquisición que, a quienes aprenden, no siempre les resulta posible transferir a nuevas situaciones.

\section{Tendencia 18: Énfasis en las respuestas únicas}

En paralelo con la tendencia anterior -centrada en el aprendizaje de conductas-, cuando en una clase predominan los contenidos declarativos por sobre los desempeños, se registra un porcentaje elevado de acciones docentes que promueven la literalidad, la transcripción de ideas -desde un material de lectura o a partir de explicaciones del profesor-. En este sentido, las actividades de bajo nivel de exigencia (Subsecretaría de Promoción de Igualdad y Calidad Educativa, 2011, pp. 25-27) son muy frecuentes tanto en el desarrollo de las clases como en las instancias de evaluación del aprendizaje de los estudiantes.

Tendencia 19: Desajuste entre lo planificado y lo que efectivamente ocurre en el aula

Sin contradecirnos con la tendencia en la que destacamos la claridad de las planificaciones, señalamos algunos quiebres entre los componentes en ellas enunciados y la realidad de la clase, entre proyección y acción; más precisamente, en tres aspectos:

- $\quad$ entre los objetivos planteados en el plan anual, en los que predomina el desarrollo de procesos cognitivos exigentes, y las actividades concretadas por los estudiantes en las clases observadas;

- $\quad$ entre las actividades previstas en las planificaciones de asignatura, enmarcadas en una metodología socioconstructivista de enseñanza, y las tareas que los docentes y los estudiantes realizan en las que se advierte el predominio en esa metodología de la impresión que hemos caracterizado y

- $\quad$ entre los recursos TIC planificados y los utilizados.

Las coincidencias más consistentes entre prácticas docentes de planificación y de gestión se advierten en los contenidos: los saberes explicitados en el programa y en las previsiones de clase son los que efectivamente ocupan a educadores y aprendices.

Tendencia 20: Predominio de los contenidos por sobre las capacidades en el quehacer de la clase

En conexión clara con la tendencia anterior, en la que destacamos la estabilidad de los contenidos en la planificación y en la acción docente, y aun cuando "en los papeles" se da un lugar preponderante a las capacidades de construir por parte de los estudiantes, en las clases reales importa más el recuerdo de conceptos que "la actuación eficaz en un tipo definido de situación, capacidad que se apoya en conocimientos pero no se reduce a ellos" (Perrenoud, 2006, p. 7).

Tendencia 21: Poco aprovechamiento de lo que saben los estudiantes

Quienes aprenden cuentan con recursos cognitivos por su condición de sujetos sociales; frente a un problema, pueden expresar muchísimas explicaciones si se les da la ocasión de hacerlo. Pero esta consideración de los recursos cognitivos de los estudiantes no es preponderante: no se suele trabajar sobre estos saberes previos para detectar posibilidades o errores ni para, a partir de ellos, propiciar redescripciones más estables, mejor informadas y más coherentes con las explicaciones de las distintas ciencias frente a un problema. En conexión estrecha con este rasgo, ponemos en foco 
cierta confusión de los docentes entre los conocimientos previos de los estudiantes y los contenidos trabajados en clases anteriores ${ }^{10}$.

Tendencia 22: Linealidad en la organización de los contenidos a enseñar en la clase

Si bien el predominio de este rasgo es distinto según el campo de contenidos considerado y hemos reconocido los esfuerzos docentes generalizados por conectar una clase con las precedentes, queda mucho por hacer en cuanto a la implementación de estrategias de enseñanza que permitan a los estudiantes la recuperación de sus conocimientos - no ya de los contenidos enseñados-, de modo de volver a ellos para, espiralada y recursivamente, promover su integración con saberes nuevos trabajados a lo largo de la unidad didáctica.

Tendencia 23: Falta de promoción del uso de la carpeta personal del estudiante como memoria de los aprendizajes construidos

Aun cuando los docentes propician enfáticamente el registro de aspectos clave del contenido en las carpetas o cuadernos -en general, su copia literal-, es escasamente suscitada su consulta como modo de entramar saberes o de buscar continuidades o diferenciaciones.

Tendencia 24: Apelación a la necesidad de aprobar como forma de explicar por qué un contenido es importante

Si bien los porcentajes de presencia de esta explicación en las clases observadas son bajos, resulta un desafío que cada docente pueda plantear por qué es importante el contenido que los estudiantes están aprendiendo sin acudir a esta estrategia de persuasión.

Tendencia 25: Concepción del involucramiento de los estudiantes como un rasgo distintivo de responsabilidad personal con el que deben contar quienes aprenden

Se registra un planteo dominante respecto de que los estudiantes llegan a la clase sin interés por aprender. Minoritariamente, se concibe este compromiso como la tarea -en la bibliografía actual, suele nombrárselo como engagement estudiantil (Zabalza, 2017) para establecer una diferencia con el interés por los contenidos, ya que no se trata solo de este- en términos de construcción que sucede por acción del docente y no como un requisito previo que el estudiante debe tener por su condición de sujeto que aprende.

Tendencia 26: Homogeneidad de tareas

Las actividades que los docentes proponen realizar a los estudiantes suelen ser iguales para todos los miembros de la clase, con pequeños márgenes de autonomía en la toma de decisiones sobre un aspecto acotado, particular de la actividad.

\footnotetext{
${ }^{10}$ Es esclarecedora la diferenciación que plantea Cols (2011): los contenidos o saberes "son enunciados proposicionales asociados de forma relativamente estable a representaciones sobre el mundo. Son objeto de reconocimiento y control social (...) Los conocimientos, en cambio, remiten a estados mentales que son el producto de experiencias cognitivas de relación entre sujetos y objetos, y entre sujetos y enunciados..." (p. 75). "Un tema simplemente recorta una porción de la realidad, más exactamente: una parte de nuestro conocimiento acerca de ella. Un tema se convierte en contenido en la medida en que se especifica en relación con ciertos propósitos formativos y se define una forma de presentación" (p. 88).
} 
Tendencia 27: Dificultades en brindar orientaciones profundas a los estudiantes

En nuestra investigación, detectamos la presencia constante de los docentes proveyendo ayudas a quienes aprenden; de hecho, hemos destacado esta tendencia. Pero, más allá de esta disponibilidad y del ofrecimiento y provisión de orientaciones, advertimos la presencia de dispositivos débiles para la evaluación formativa. Por ejemplo: preguntar si todo ha quedado claro; ante errores, modelizar cuál es una respuesta aceptable; centrarse en ayudas para que el estudiante supere una dificultad puntual, sin acompañarlo a ahondar en las razones de su error; predominantemente, proveer asesoramiento a los estudiantes que expresan dificultades (cuando solo $3 \%$ del total lo hace), por lo que estas decisiones garantizan que las ayudas resulten eficaces.

Tendencia 28: Falta de generalización de estrategias didácticas que permitan la construcción de aprendizajes por parte de todos los estudiantes

En las clases, se registra un porcentaje de estudiantes (del 7 al $22 \%$, según el campo de contenidos considerado) cuya participación no se logra a partir de las estrategias de enseñanza que el docente ha previsto y que se encuentra implementando. La idea de "todos aprendiendo" aún no es una realidad.

Tendencia 29: Aprovechamiento de recursos audiovisuales circunscripto a la provisión de información y a su recuerdo

Si bien destacamos favorablemente la presencia de materiales para la enseñanza y el aprendizaje, aún no resulta predominante el uso de estos recursos como promotores de pensamiento, como herramientas auxiliares de una investigación asumida por los estudiantes.

Tendencia 30: Insuficiente integración de TIC en las clases en relación con otros recursos didácticos

Los recursos digitales se integran en no más del $17 \%$ de las clases observadas con pequeñas diferencias entre áreas (aunque el $80 \%$ de los docentes ha planificado su uso). Superar este límite constituye un desafío, así como integrar las TIC no solo para presentar información a ser vista y escuchada por los estudiantes, sino en tanto auxiliares de procesos de comprensión, modelización e investigación, es decir, comprometidas con aprendizajes exigentes. Como parte de esta tecnología digital vacante, se encuentran los teléfonos celulares y los dispositivos móviles con que los estudiantes cuentan, los que son escasamente integrados con fines educativos a pesar de su disponibilidad.

Tendencia 31: Uso parcial del equipamiento disponible en las escuelas

Las instituciones educativas consideradas en el estudio cuentan con recursos didácticos que no se integran en las clases.

Tendencia 32: Escasas ocasiones de coevaluación

Si bien hemos advertido diferencias importantes según el área curricular considerada, los procesos de evaluación del aprendizaje de los estudiantes quedan, prioritariamente, a cargo de los docentes, sin considerarse la evaluación entre pares como dispositivo formativo. 


\section{Una vuelta a las hipótesis del estudio}

En el primero de nuestros documentos, Plan de trabajo (2015), planteamos las hipótesis a investigar en Las prácticas de enseñanza de los docentes de educación secundaria. Un estudio en las provincias de Córdoba, Buenos Aires y Entre Ríos, República Argentina (2016-2018). Estas son las siguientes:

1. "las prácticas de enseñanza no siempre ponen de manifiesto el sustento teórico que, ya sea de manera expresa o implícita, el docente sostiene como marco de referencia;

2. parecen coexistir prácticas de enseñanza que responden a enfoques y concepciones de enseñanza más tradicionales con otras que enlazan con propuestas más innovadoras y renovadas;

3. cierta desvalorización del docente no solo respecto de sí mismo, en cuanto al propio rol, sino también en relación con sus desempeños y vínculos con sus pares y con los estudiantes;

4. la existencia de una distancia entre el diseño de las prácticas y su gestión". (p. 16)

Desarrollados todos los estudios previstos a lo largo de estos años de investigación, se confirman la primera, la segunda y la cuarta y se desestima la tercera hipótesis, ya que no contamos con datos que nos permitan comprobarla, argumentar acerca de tal desvalorización ni concluir al respecto. En nuestras entrevistas realizadas a 2111 profesionales (2019 b), solo un $4 \%$ de los colegas expresa que el docente de escuela secundaria es un profesional "despreciado", "desacreditado", "ignorado" y un porcentaje equivalente lo pone de manifiesto en los autorreportes.

\section{Para cerrar esta presentación}

Nos propusimos desarrollar una investigación respetuosa de lo que los docentes son y hacen y hemos logrado concretarla. Nos distanciamos conscientemente de lo que los investigadores Ilaman validez nominal -aceptación de características sin someterlas a revisión profunda-, en este caso, referida a algunos rasgos con que suele asociarse la educación secundaria argentina, y logramos problematizar esos juicios buscando evidencia empírica que los validara o que los excluyera. Rechazamos toda forma de erosión o de desvalorización de la profesión docente, resguardo ético que ha estado presente en cada ítem de nuestras encuestas, en cada pregunta de nuestras entrevistas, en cada componente de nuestro protocolo de observación de clases. A lo largo de estos tres años de tarea, hemos mantenido nuestro posicionamiento democrático, dialogante y crítico, cuidadoso y responsable, lo que nos hace estar por completo satisfechos del proceso de investigación asumido y concretado.

Finalmente, hemos respaldado las tendencias aquí expresadas con los datos que pueden encontrarse en los documentos puntualizados y con nuestros argumentos respecto de ellos, por lo que cerramos este artículo del mismo modo con el que lo abrimos: invitando a la lectura de esos documentos, porque son los que dan cuenta de la confiabilidad de nuestros planteos y porque permiten el acceso a información más detallada. 


\section{Documentos producidos en el marco de la investigación}

Ferreyra, H. y Di Francesco, A. (dir.) (2015). Plan de trabajo. Las prácticas de enseñanza de los docentes de educación secundaria. Un estudio en las provincias de Córdoba, Buenos Aires y Entre Ríos, República Argentina (2016-2018). Córdoba, Argentina: Equipo de Investigación de Educación de Adolescentes y Jóvenes de la Facultad de Educación de la Universidad Católica de Córdoba.

Ferreyra, H. y Rúa, A. (2016 a). Conceptos que dan marco a esta investigación. Córdoba, Argentina: Equipo de Investigación de Educación de Adolescentes y Jóvenes de la Facultad de Educación de la Universidad Católica de Córdoba.

Ferreyra, H. (2016 b). Dimensiones, categorías y aspectos a estudiar. Córdoba, Argentina: Equipo de Investigación de Educación de Adolescentes y Jóvenes de la Facultad de Educación de la Universidad Católica de Córdoba.

Ferreyra, H. (2016 c). Planificación de la encuesta virtual. Córdoba, Argentina: Equipo de Investigación de Educación de Adolescentes y Jóvenes de la Facultad de Educación de la Universidad Católica de Córdoba.

Ferreyra, H. (2016 d). Guía para la observación de clases. Córdoba, Argentina: Equipo de Investigación de Educación de Adolescentes y Jóvenes de la Facultad de Educación de la Universidad Católica de Córdoba.

Ferreyra, H. (2017 a). ¿Qué es lo que ocurre en nuestras aulas de secundaria? Ensayos referidos a la educación secundaria argentina. Córdoba, Argentina: Editorial de la Universidad Católica de Córdoba. Recuperado de http://pa.bibdigital.uccor.edu.ar/1383/1/ensayos\%20\%281\%29.pdf.

Ferreyra, H. (2017 b). Mirar para aprender en contexto. Observación de clases. Córdoba, Argentina: Editorial de la Universidad Católica de Córdoba. Recuperado de http://pa.bibdigital.uccor. edu.ar/1477/1/Ferreyra.informe\%20UCC.pdf.

Ferreyra, H. y López, M. (coord.) (2017 c). Educación secundaria: Prácticas de enseñanza en foco. Córdoba, Argentina: Editorial de la Universidad Católica de Córdoba. Recuperado dehttps:// equipodeinvestigacion2014ayj.com/2017/11/21/album-de-fotos-y-videos-con/.

Ferreyra, H. y Bono, L. (coord.) (2018 a). Segundo Foro y Seminario. Debates sobre el currículum de Ciencias Naturales de la Educación Secundaria en relación con las prácticas: avances, retos y desafíos de cara al futuro. Algunas propuestas para orientar la educación en Ciencias Naturales en la Argentina hacia el 2030, poniendo el foco en las prácticas. Informe final. Córdoba, Argentina: Editorial de la Universidad Católica de Córdoba. Recuperado de https://equipodeinvestigacion2014ayj.com/2018/06/05/publicacion-debates-sobre-el-curriculum-de-ciencias-naturales-de-la-educacion-secundaria-en-relacion-con-las-practicas/.

Ferreyra, H. (2018 b). Debates sobre el currículum de Ciencias Sociales y Humanidades en la Educación Secundaria, en clave de las prácticas educativas que miran los tiempos presentes y por-venir. Informe final. Córdoba, Argentina: Editorial de la Universidad Católica de Córdoba. Recuperado de https://equipodeinvestigacion2014ayj.com/2018/05/31/publicacion-debates-sobre-el-curriculum-de-ciencias-sociales-y-humanidades-en-la-educacion-secundaria/.

Ferreyra, H. y Molinolo, S. (coord.) (2018 c). Debates sobre el currículum de Matemática en la educación secundaria: avances y desafíos en relación con las prácticas de enseñanza, de cara al futuro. Informe final. Córdoba, Argentina: Editorial de la Universidad Católica de Córdoba. Recuperado de https://equipodeinvestigacion2014ayj.com/2018/06/09/seminario-debates-sobre-el-curriculum-de-matematica-en-la-educacion-secundaria-avances-y-desafios-en-relacion-con-las-practicas-de-ensenanza-de-cara-al-futuro/. 
Ferreyra, H. y García García, N. (comp.) (2018 d). Dimensión ética. Córdoba, Argentina: Equipo de Investigación de Educación de Adolescentes y Jóvenes de la Facultad de Educación de la Universidad Católica de Córdoba.

Ferreyra, H. y Vázquez, M. (comp.) (2018 e). Ventanas Johari sobre prácticas docentes. Córdoba, Argentina: Equipo de Investigación de Educación de Adolescentes y Jóvenes de la Facultad de Educación de la Universidad Católica de Córdoba.

Ferreyra, H. (2018 f). Dimensiones involucradas en el estudio de las prácticas de enseñanza. Revista Educación y Humanismo, 20 (34), 128-141. Recuperado de http://revistas.unisimon.edu. co/index.php/educacion/article/view/2861.

Ferreyra, H. (2018 g). Las prácticas de enseñanza como dimensión constitutiva de la calidad de la educación. Revista Pertinencia Académica, 8 (sept.), 101-124. Babahoyo, Ecuador: Vicerrectorado Académico, Universidad Técnica de Babahoyo. Recuperado de http://revista-academica. utb.edu.ec/index.php/pertacade/article/view/132.

Ferreyra, H. (2018 h). Gacetillas de prensa. Córdoba, Argentina: Equipo de Investigación de Educación de Adolescentes y Jóvenes de la Facultad de Educación de la Universidad Católica de Córdoba.

Ferreyra, H. (2018 i). Rueda del aprendizaje escolar. Recurso didáctico. Córdoba, Argentina: Editorial de la Universidad Católica de Córdoba y Editorial Comunicarte. Recuperado de http:// horacioaferreyra.com.ar/wp-content/uploads/2019/03/RUEDA-APRENDIZAJE-fusionado.pdf.

Ferreyra, H. (2019 a). Las prácticas de enseñanza de los docentes de Educación Secundaria a través de encuestas. Córdoba, Argentina: Editorial de la Universidad Católica de Córdoba. Recuperado de http://horacioaferreyra.com.ar/wp-content/uploads/2019/03/PA-ENCUESTAS.pdf.

Ferreyra, H. (2019 b). Las prácticas de enseñanza de los docentes de Educación Secundaria a través de observaciones de clases, planificaciones didácticas y entrevistas. Córdoba, Argentina: Editorial de la Universidad Católica de Córdoba. Recuperado de http://horacioaferreyra.com.ar/ wp-content/uploads/2019/03/PA-ENTREVISTAS.pdf.

Ferreyra, H. (2019 c). Enseñar en Educación Secundaria, un compromiso con la construcción de aprendizajes relevantes y duraderos. Secuencias didácticas implementadas en los distintos ciclos y años de la Educación Secundaria y Modalidades. Córdoba, Argentina: Editorial de la Universidad Católica de Córdoba. Recuperado de http://horacioaferreyra.com.ar/wp-content/ uploads/2019/03/sec-css-y-hum.pdf, http://horacioaferreyra.com.ar/wp-content/uploads/2019/ 03/sec-leng-y-com.pdf y http://horacioaferreyra.com.ar/wp-content/uploads/2019/03/secuencias-cs-nat-mate.pdf.

Ferreyra, H. Bono, L. y Brito, G. (coord.) (2019 d). Las prácticas de enseñanza en la educación secundaria I. Córdoba, Argentina: Equipo de Investigación de Educación de Adolescentes y Jóvenes de la Facultad de Educación de la Universidad Católica de Córdoba.

Ferreyra, H. y Bono, L. (coord.) (2019 e). Las prácticas de enseñanza en la educación secundaria II. Córdoba, Argentina: Equipo de Investigación de Educación de Adolescentes y Jóvenes de la Facultad de Educación de la Universidad Católica de Córdoba.

\section{Referencias bibliográficas}

Becker, H. (2018). Datos, pruebas e ideas. Buenos Aires: Siglo Veintiuno.

Cols, E. (2011). Estilos de enseñanza. Rosario, Argentina: Homo Sapiens. 
Ferreyra, H. (dir.) (2015). La calidad de la Educación Secundaria: La escuela posible como horizonte de expectativas. Córdoba, Argentina: Equipo de Investigación de Educación de Adolescentes y Jóvenes de la Facultad de Educación de la Universidad Católica de Córdoba. Recuperado el 12 de octubre de 2020 de: http://pa.bibdigital.uccor.edu.ar/696/1/PREMIO\%20SARMIENTO\%20 UNICEF.pdf.

Kemmis, S. (1996). La teoría de la práctica educativa. En: Carr, W. Una teoría para la educación. Hacia una investigación educativa crítica. Madrid: Morata.

Meirieu, P. (2016). Recuperar la pedagogía. De lugares comunes a conceptos clave. Buenos Aires: Paidós.

Otero, M. (2018). Mantener la atención de los chicos, uno de los mayores desafíos docentes. La Voz. Córdoba, Argentina. Recuperado el 20 de octubre de 2020 de: https://www.lavoz.com.ar/ ciudadanos/mantener-atencion-de-chicos-uno-de-mayores-desafios-docentes.

Perrenoud, Ph. (2006). Construir competencias desde la escuela. Santiago de Chile: J. C. Sáez.

Stake, R. (2006). Evaluación comprensiva y evaluación basada en estándares. Barcelona, España: Graó.

Subsecretaría de Promoción de Igualdad y Calidad Educativa. Secretaría de Educación. Ministerio de Educación de la provincia de Córdoba (2011). La evaluación de aprendizajes en la escuela secundaria. Córdoba, Argentina: Subsecretaría de Promoción de Igualdad y Calidad Educativa. Recuperado el 20 de octubre de 2020 de: http://www.igualdadycalidadcba.gov.ar/SIPEC-CBA/ publicaciones/Capac\%20Nivel\%20Secundario/Documento\%20Evaluacion\%20Secundaria\%20 21-10-11.pdf.

UCC (2011). Proyección y responsabilidad social universitaria. Córdoba, Argentina: Universidad Católica de Córdoba. Recuperado el 20 de octubre de 2020 de: https://www.ucc.edu.ar/proyeccion/secretaria-de-proyeccion-y-responsabilidad-social-universitaria/.

Zabalza, M. (2017). Las buenas prácticas docentes y su impacto en el engagement estudiantil. Buenos Aires: Academia Nacional de Educación. 